\title{
Repetitive Project Scheduling Optimization with Soft Logic Considering Maximum Constraints and Resource Continuity Constraints
}

\author{
Feng Kong ${ }^{1}$ Chen Yuan ${ }^{2, *}$

\begin{abstract}
${ }^{1}$ Prof.School of Economics and Management, North China Electric Power University, Baoding, Hebei 071000, China
${ }^{2}$ MS.School of Economics and Management, North China Electric Power University, Baoding, Hebei 071000, China
\end{abstract} \\ *Corresponding author. Email: 2182218061@ncepu.edu.cn
}

\begin{abstract}
Project scheduling is an essential branch of construction management, which has been proved of practical worth. This paper aims to make improvements in it by adding new constraint to this problem to make it more practical. This article firstly establishes a model which can be optimized with soft logic for repetitive projects under the constraint of resource continuity. After model being established, the maximum and minimum constraints that constraint condition are taken into consideration to fill in the constraint condition. In this paper, a mathematical model considering soft logic is established to address the repetitive project optimization problem that coexists with maximum constraints and resource continuity constraints. The goal is to choose the appropriate construction mode and unit construction order between the units to minimize the corresponding construction cost, which aims to satisfy construction aims. Multi-objective solution is used to obtain the Pareto curve of the solution set and output multiple feasible solutions. An example of construction management that meets the hypothesis of this paper is used to prove that soft logic can optimize it.
\end{abstract}

Keywords: project management, soft logic, minimum and maximum constraints, repetitive project scheduling, genetic algorithm

\section{INTRODUCTION}

The development of project management can be traced back to the beginning of the 20th century. The Gantt chart invented by Gantt in 1917 used horizontal progress bars to represent the duration of each process and put vertical labels to mean the specific process, which forms a schematic diagram that can intuitively reflect different activities and their duration. Construction management has been developed to a practical discipline and been widely applied in projects. CPM is an important solution to the problem of project management.

Since the 21th century, the time-cost trade-off problem (TCTP) has attracted widespread attention. Many researchers make improvements on the model of project management problems, mainly including the establishment of objective functions and the setting of constraints. Current researches on schedule control in the field of project management are mostly about time-cost trade-offs (TCTP) problem, which is considered as the NP-hard problem. In the traditional TCTP problem, duration of each activity is a fixed number, and there is a certain logical relationship between those activities, but it can not meet actual project management.

Constraints in network planning include maximum time constraints, minimum time constraints, and probability time constraints, etc. The kind of maximum time constraint construction problems are extensively used, especially in civil engineering construction management. The coating of sticky layer oil in asphalt road construction is a good example, in which the time from the transport to the conveyance of the concrete must not exceed 25 degrees centigrade within 240 minutes. There is a maximum time constraint of 240 minutes in the construction of this project.

Correspondingly, There are sometimes some minimum time constrained logical relationships between these different procedures. For example, the precondition for laying out the concrete after pouring is that the 
strength of the concrete surface must reach $5 \mathrm{MPa}$. Therefore, construction cannot be performed immediately after the concrete is poured, and construction can be performed after standing for a period of time.The minimum constraint and the maximum constraint exist widely in engineering projects, which are closely related not only to the project schedule, but also to the technical requirements of great importance to the quality of the project, which has been proved to have practical worth, especially in the field of project management.

\section{LITERATURE REVIEW}

For repetitive projects management, techniques are available for performing different optimization procedures at the initial scheduling stage but they perform schedule acceleration.Repetitive construction projects can be optimized when considering soft logic. Heravi, G. considered an effective model for repetitive construction project scheduling and met the actual scheduling needs. This model combines the newly developed repetitive activity resource-driven scheduler, repetitive construction scheduling optimizer, and integration of repetitive and non-repeating scheduling technologies. The optimization of this model is $\mathrm{TCTP}^{[1]}$.

The algorithms to solve the problems of network planning are various. A branch-and-bound algorithm for a class of single scheduling problems with minimum and maximum time delays was proposed(Gwo-Ji Sheen $2007)^{[2]}$, which has a certain reference value for the minimum and maximum constraints in this paper. Xin Zou (2020) proposed a non-typical scheduling constraint programming method for repetitive projects with soft logic, and improved genetic algorithm to solve the problem of this project, and integrated the soft logic into the time cost trade-off and established a flexible repetitive scheduling model. This model allows the same activities to be performed in parallel in different units ${ }^{[3]}$.Wang (2005) simulates the duration of an example project by considering uncertain activity durations and random soft links ${ }^{[4]}$.

Research of time lags are divided as follows. Sheen and Liao (2007) Studies completed in the minimum time lag and the maximum completion time lag of single scheduling problems. Using the branch and bound algorithm is proposed to find the optimal scheduling in citation [2]. Kreter, Schutt and Stuckey (2017) considered time constraints and calendar constraints in RCPSP problems and developed six different Constraint Programming (CP) model to solve this problem ${ }^{[5]}$. YongSheng, Chang-Yong, and Li-Ying (2010) propose a discrete time cost trade-off is a multi-objective problem often encountered in project management ${ }^{[6]}$. Linear timecost trade-off problem can be solved in polynomial time, but its discrete form is np- difficult(Skutella 1997) ${ }^{[7]}$.

\section{PROBLEM FORMULATIONS}

Soft logic often exists in repetitive projects. Because in repetitive projects,it is available to make sequence adjustments to some processes of a project. However, it will not have a bad impact on the entire project, it just changes the overlapping relationship of the process. For example, there are 5 buildings in a project that need to be built, and the processes for each building are the same. It can be performed according to the fixed logic of $1 \rightarrow 2 \rightarrow 3 \rightarrow 4 \rightarrow 5$, or it can be performed according to soft logic. The model in this paper considers maximum constraints and resource continuity constraints. Repetitive projects with soft logic that can address this constraint.

The repetitive item contains $\mathbf{M}$ activities, numbered $\mathrm{i}=1,2,3, \ldots \mathrm{M}$. Each item has $\mathrm{N}$ repetitive units, numbered $\mathrm{j}=1,2,3, \ldots \mathrm{N}$. Activity 1 is the start process and Activity $\mathrm{M}$ is the end process. This sequence will not change. A certain active process $i$ has connection with its previous activity $q \cdot \mathbf{q} \in \mathbf{Q}_{i}$ and $Q_{i}$ is a set of all the preceding activities of activity $i$.Each activity must be executed in a given mode of $L_{i}$, but if one mode has been selected, all units must be completed in this mode. Considering soft logic in this article, the construction order of repetitive units in this article can be changed, but the determined construction order must be applied to all activities and $\mathrm{i}=1,2,3, \ldots \mathrm{M}$.

\subsection{Objective function}

There are three types of construction costs in this article. Direct costs, indirect costs, and transshipment costs. Direct cost includes the funds used for each job to adopt a different construction method. Indirect cost refers to the cost to be used on the construction in one selected mode per day, which is fixed. Besides, in the case of this paper, the model of FTS = transit time is adopted, which is called the constraint of resource continuity. This article does not take idle cost of resources into consideration and it is decided by the mathematical model which is suitable for the construction with high idle cost of some resources. Because of the resource continuity, resource idle cost is included in the cost of resource transferring without additional calculation. In the mathematical model, resource continuity is a constraint that must be met. The last part is the resource transferring cost. Resources transferring require time and costs. The cost can be calculated according to the actual situation.

The objective function of this paper is the minimization of costs. The objective function is to minimize the total cost.

$$
\text { Minimize } \quad \mathrm{TC}=\sum_{\mathrm{i}=1}^{\mathrm{M}}\left(\mathrm{DC}_{\mathrm{i}}+\mathrm{RTC}_{\mathrm{i}}\right)+\mathrm{IC}
$$




$$
\begin{aligned}
& \mathrm{DC}_{\mathrm{i}}=\sum_{\mathrm{k}=1}^{\mathrm{ki}} \sum_{\mathrm{j}=1}^{\mathrm{N}} \mathrm{X}_{\mathrm{ik}} \mathrm{d}_{\mathrm{ijk}} \mathrm{C}_{\mathrm{ik}} \\
& X_{i k}=\left\{\begin{array}{l}
1, \text { mode of } \mathrm{i}=\mathrm{k} \\
0, \text { else }
\end{array}\right. \\
& \mathrm{RTC}=\sum_{\mathrm{k}=1}^{\mathrm{Ki}} \sum_{\mathrm{j} 1=1}^{\mathrm{N}} \sum_{\mathrm{j} 2=1}^{\mathrm{N}} \mathrm{X}_{\mathrm{ik}} \mathrm{y}_{\mathrm{j} 1 \mathrm{j} 2 \mathrm{r}} \mathrm{r}_{\mathrm{j} \mathrm{j} 2}^{\mathrm{k}} \\
& y_{j 1 . j_{2}}=\left\{\begin{array}{l}
1, \text { if } j_{2} \text { is scheduled to start after } j_{1} \\
0, \text { else }
\end{array}\right. \\
& \mathrm{IC}=\mu \mathrm{D}_{\mathrm{p}}
\end{aligned}
$$

Equation (2)Represents the total cost of executing each unit in a certain process in a certain process in a certain process.Equation (3) means that only a certain process is in mode $\mathrm{k}$, its value is 1 , otherwise it is 0 . $\mathrm{d}_{\mathrm{ijk}}$ means the time required for step $i$ in unit $j$ to proceed in mode $\mathrm{k}$ cik means the daily direct cost of process $\mathrm{i}$ in mode $\mathrm{k}$.Equation (4) represents the cost required to perform the transfer in a unit under construction in a certain process when performing a construction transfer. The resource transfer process is relatively short. This article intends to consider the cost of resource transfer, including the cost of maintaining the resource. Since this model is built for materials with large idle cost of resources, this model guarantees the continuity of resources, so the total cost does not include the item of idle cost of resources.Equation (5) represents that it is equal to 1 only when $\mathrm{j}_{2}$ is planned to be performed after $\mathrm{j}_{1}$, or to 0 otherwise. $\mathrm{r}_{\mathrm{j} \cdot \mathrm{j} 2}^{\mathrm{k}}$ represents the cost required for resource transfer between $\mathrm{j}_{1}$ and $\mathrm{j}_{2}$ in mode k.Equation (6) Represents the cost required for resource transfer between $\mathrm{j}_{1}$ and $\mathrm{j}_{2}$ in mode $\mathrm{k}$.

\subsection{Set of Mathematical Model}

Soft start time of an active logical constraints require each cell is bigger than a time before completion of the step unit. (If this event is not the first case of a process) at the same time, for a particular process, the need to ensure continuity of resources. Soft logic resource constraints continuous case can be represented by a mathematical model as follows and binary variables $j_{1} j_{2}$ need to meet the following constraints.

$$
\begin{aligned}
& \sum_{j 1=1}^{N} \sum_{j 2=1}^{N} y_{j i j 2}=N-1 \\
& \sum_{j 1=1}^{N} y_{j i j 2} \leq 1, j_{2}=1,2, \ldots N \\
& \sum_{j 2=1}^{N} y_{j i j 2} \leq 1, \quad j_{1}=1,2, \ldots N
\end{aligned}
$$

$$
\sum(\mathrm{u}, \mathrm{v}) \in \mathrm{yuv} \leq|\mathrm{V}|-1, \mathrm{~V} \subset\{1,2, \ldots \mathrm{N}\}, 2 \leq|\mathrm{V}| \leq \mathrm{N}-1(10)
$$

Equation (7) indicates that for a certain construction unit, the number of times the resources are transferred in the process and process; (8), (9) indicates that the number of times that resources can be transferred in any two construction units can only be at most 1, that is, transportation cannot be repeated.

\subsubsection{Priority Relationship Constraints}

There are two types of constraints on precedence relations in this paper. First, for the construction of repetitive projects, soft logic is considered, and the constraint of the FTS overlap relationship is FTS = resource transfer time. That is, the requirement to ensure the continuity of resources. This constraint can be expressed as: if unit $\mathrm{j} 2$ follows unit $\mathrm{j} 1$, then:

$$
\begin{aligned}
& S_{i j 2}=S_{i j 1}+\sum_{k=1}^{K_{i}} X_{i k}\left(d_{i j i k}+r_{j i j 2}^{k}\right) \\
& \text { FTS }=[a, b] \\
& S_{i i j}+\sum_{k=1}^{K_{i}} X_{i k} * d_{i j j k}+b \geq S_{i 2 j} \geq S_{i j j}+\sum_{k=1}^{K_{i}} X_{i k} * d_{i j k}+a \\
& S_{i 2 j} \geq S_{i i j j}+\sum_{k=1}^{K_{i}} X_{i k} * d_{i j j k}+a \\
& \sum_{k=1}^{K_{i}} X_{i k}=1, i=1,2, \ldots M
\end{aligned}
$$

Equation (12) shows that there are maximum and minimum constraints in the construction of any one of the two adjacent processes and that the next process must be performed within the range of $[a, b]$ after the previous process is completed: if a process is followed by a process, then any unit has the following constraints as shown in Equation (13).For operations without minimum and maximum constraints, if the operation is followed immediately by the operation, there are shown in Equation (14).As each process is set in this article, only one mode is allowed. Therefore, the repetitive items in this article should correspond with Equation (15).

\subsubsection{Coding of genetic algorithms}

The coding of a repetitive projects combines of a pair of number lists. The project example used in Case 2 of this article contains six units, and each unit has five processes to complete. One chromosome of length 11 . The first half of the chromosome is six digits and the second half is five digits. The first half of the chromosome indicates the soft logic construction sequence of the project(the $i_{\text {th }}$ gene in the.The last five digits indicate the execution mode of the project's five procedures. 
Step 1: Crossover. A chromosome is 11 bits in length, and the intersection point that can be crossed is at any position of the last five nodes. The crossover diagrams are shown below. The first half of the cross section is unchanged, and the second half is exchanged.

Step2: Variation.For each of the 11chromosomes, mutation may occur in each chromosome. If a mutation occurs in one of the first six digits, the mutated number should be replaced with the previous number. Otherwise, the construction logic is not satisfied. When the last five digits are in the operation of mutation, the mutation becomes another number within the scope of the pattern.

\section{EXAMPLE ANALYSIS}

This article intends to give a practical example of concrete construction based on actual construction operations.

In the actual concrete operation, the maintenance cost of the concrete that has been formed by mixing is relatively high. Therefore, in the construction of this unit, the overlap method used for the same process (because the transportation time is essential, it is not considered that the constraint condition of the continuity of resources is destroyed). This paper intends to adopt a mathematical model for the constrained maximum continuity of soft logic resources for repetitive projects. And this model is applied to solve the practical problems of multiple concrete repetitive projects.

There are five steps in concrete construction defined as Activity 1,2,3,4,5. Activity 1 represents construction preparations to be done, including excavation and site cleaning up. Activity 2 implies construction concrete mixing and transportation (pumping). In order to be fully stirred, the implementation of this process in each building must take more than a certain time, but if the initial setting time is exceeded, the quality of the concrete is unqualified and cannot meet the requirements of construction. In the project, there are both maximum and minimum constraints. Activity 3 indicates construction pouring concrete columns and pouring beams and slabs. Activity 5 indicates carrying out concrete curing. In the construction of this project, because the idle cost of resources such as concrete is high, this paper takes it into consideration. In each process, FTS $=0$ must be guaranteed between the buildings. The transit time between the two buildings should be as short as possible in order to reduce total cost.

Besides, there are some constraints in this case. Firstly, the constraints adopted in this example were shown between steps 2 to 3 . Secondly, because the cost of process transfer is more diversified, in general, the transfer process selected by the operation is generally as short as possible in the order of transfer, rather than in the order of long transfer time. Thirdly, the daily indirect cost of construction in this case is 200 yuan.In the following tables, B1 to B6 represent Building 1 to Building 6.

The population size to be used in the calculation in this paper is 200 , the maximum iteration number is 200 , the crossover probability is 0.8 , and the mutation probability is 0.3 .

The calculation results obtained are shown below, and the corresponding construction order and mode selection are shown in TABLE3.

Table 1. Project Data

\begin{tabular}{l|l|l|l|l|l|l|l|l}
\hline & Mode & IC & B1 & B2 & B3 & B4 & B5 & B6 \\
\hline \multirow{2}{*}{ Activity 1 } & 1 & 100 & 4 & 6 & 5 & 6 & 10 & 8 \\
\cline { 2 - 9 } & 2 & 400 & 2 & 3 & 2.5 & 3 & 5 & 4 \\
\hline \multirow{2}{*}{ Activity 2 } & 1 & 200 & 12 & 10 & 14 & 8 & 6 & 8 \\
\cline { 2 - 9 } & 2 & 400 & 9 & 7.5 & 10.5 & 6 & 4.5 & 6 \\
\cline { 2 - 9 } & 3 & 800 & 6 & 5 & 7 & 4 & 3 & 4 \\
\hline
\end{tabular}

Time lags Activity 2 and Activity 3 FTS=[2,7]

\begin{tabular}{l|l|l|l|l|l|l|l|l}
\hline Activity3 & 1 & 300 & 8 & 6 & 6 & 4 & 5 & 4 \\
\cline { 2 - 9 } & 2 & 600 & 6 & 4.5 & 4.5 & 3 & 4 & 3 \\
\cline { 2 - 9 } & 3 & 1200 & 4 & 3 & 3 & 2 & 2.5 & 2 \\
\hline \multirow{4}{*}{ Activity4 } & 1 & 200 & 10 & 12 & 8 & 14 & 10 & 8 \\
\cline { 2 - 9 } & 2 & 400 & 7.5 & 8 & 6 & 10.5 & 7.5 & 6 \\
\cline { 2 - 9 } & 3 & 800 & 5 & 6 & 4 & 7 & 5 & 4 \\
\hline Activity5 & 1 & 150 & 6 & 4 & 5 & 3 & 4 & 3 \\
\hline
\end{tabular}




\begin{tabular}{|l|l|l|l|l|l|l|l}
2 & 300 & 4.5 & 3 & 4 & 2 & 3 & 2 \\
\hline 3 & 600 & 3 & 2 & 3 & 1.5 & 2 & 1.5 \\
\hline
\end{tabular}

Table 2. Resource-transferring time and Resource-transferring cost ( RTC ) in Activity 1,2,3,4, 5.

\begin{tabular}{l|l|l|l|l|l|l|l|l|l|l|l|l|l}
\multicolumn{1}{l}{ In Activity 1,3,5. } & \multicolumn{1}{l|}{ In Activity 2,4. } \\
\hline & B1 & B2 & B3 & B4 & B5 & B6 & & B1 & B2 & B3 & B4 & B5 & B6 \\
\hline B1 & - & $0.5,100$ & $0.5,100$ & $0.5,100$ & $0.5,100$ & 1,200 & B1 & - & 1,200 & 1,200 & 1,200 & 1,200 & 2,400 \\
\hline B2 & $0.5,100$ & - & $0.5,100$ & $0.5,100$ & 1,200 & $0.5,100$ & B2 & 1,200 & - & 1,200 & 1,200 & 2,400 & 1,200 \\
\hline B3 & $0.5,100$ & $0.5,100$ & - & 1,200 & $0.5,100$ & $0.5,100$ & B3 & 1,200 & 1,200 & - & 2,400 & 1,200 & 1,200 \\
\hline B4 & $0.5,100$ & $0.5,100$ & 1,200 & - & $0.5,100$ & $0.5,100$ & B4 & 1,200 & 1,200 & 2,400 & - & 1,200 & 1,200 \\
\hline B5 & $0.5,100$ & 1,200 & $0.5,100$ & $0.5,100$ & - & 1,200 & B5 & 1,200 & 2,400 & 1,200 & 1,200 & - & 2,400 \\
\hline B6 & 1,200 & $0.5,100$ & $0.5,100$ & $0.5,100$ & 1,200 & - & B6 & 2,400 & 1,200 & 1,200 & 1,200 & 2,400 & - \\
\hline
\end{tabular}

TABLE 3. Operation result of matlab.

\begin{tabular}{|c|c|c|c|c|c|c|c|c|c|c|c|c|c|c|c|c|c|}
\hline \multirow{2}{*}{$\frac{\text { No. }}{1}$} & \multirow{2}{*}{$\begin{array}{l}\text { Unit construction } \\
\text { sequence } \\
4 \rightarrow 2 \rightarrow 1 \rightarrow 5 \rightarrow 3 \rightarrow 6\end{array}$} & \multicolumn{5}{|c|}{$\begin{array}{l}\text { Selected } \\
\text { mode }\end{array}$} & \multirow{2}{*}{$\begin{array}{l}\text { durati } \\
\text { on } \\
52.5\end{array}$} & \multirow{2}{*}{$\begin{array}{l}\text { Total } \\
\text { Cost } \\
87500\end{array}$} & \multirow{2}{*}{$\frac{\text { No. }}{20}$} & \multirow{2}{*}{$\begin{array}{l}\text { Unit construction } \\
\text { sequence } \\
5 \rightarrow 1 \rightarrow 4 \rightarrow 2 \rightarrow 3 \rightarrow 6\end{array}$} & \multicolumn{5}{|c|}{$\begin{array}{l}\text { Selected } \\
\text { mode }\end{array}$} & \multirow{2}{*}{$\begin{array}{l}\text { durati } \\
\text { on } \\
75.5\end{array}$} & \multirow{2}{*}{$\begin{array}{l}\text { Total } \\
\text { Cost } \\
79350\end{array}$} \\
\hline & & 2 & 3 & 1 & 3 & 3 & & & & & 1 & 3 & 1 & 2 & 2 & & \\
\hline 2 & $4 \rightarrow 5 \rightarrow 1 \rightarrow 2 \rightarrow 3 \rightarrow 6$ & 2 & 3 & 1 & 3 & 3 & 52.5 & 87500 & 21 & $4 \rightarrow 5 \rightarrow 1 \rightarrow 2 \rightarrow 3 \rightarrow 6$ & 1 & 3 & 1 & 2 & 1 & 76.5 & 77750 \\
\hline 3 & $4 \rightarrow 2 \rightarrow 1 \rightarrow 5 \rightarrow 3 \rightarrow 6$ & 2 & 3 & 1 & 3 & 2 & 53 & 85350 & 22 & $4 \rightarrow 5 \rightarrow 1 \rightarrow 3 \rightarrow 2 \rightarrow 6$ & 1 & 3 & 1 & 2 & 1 & 76.5 & 77750 \\
\hline 4 & $4 \rightarrow 5 \rightarrow 1 \rightarrow 2 \rightarrow 3 \rightarrow 6$ & 2 & 3 & 1 & 3 & 2 & 53 & 85350 & 23 & $5 \rightarrow 1 \rightarrow 3 \rightarrow 2 \rightarrow 4 \rightarrow 6$ & 1 & 3 & 1 & 2 & 1 & 76.5 & 77750 \\
\hline 5 & $4 \rightarrow 2 \rightarrow 1 \rightarrow 5 \rightarrow 3 \rightarrow 6$ & 2 & 3 & 1 & 3 & 1 & 54.5 & 83850 & 24 & $5 \rightarrow 1 \rightarrow 3 \rightarrow 2 \rightarrow 6 \rightarrow 4$ & 1 & 3 & 1 & 2 & 1 & 76.5 & 77750 \\
\hline 6 & $4 \rightarrow 5 \rightarrow 1 \rightarrow 2 \rightarrow 3 \rightarrow 6$ & 2 & 3 & 1 & 3 & 1 & 54.5 & 83850 & 25 & $5 \rightarrow 1 \rightarrow 4 \rightarrow 2 \rightarrow 3 \rightarrow 6$ & 1 & 3 & 1 & 2 & 1 & 76.5 & 77750 \\
\hline 7 & $4 \rightarrow 2 \rightarrow 1 \rightarrow 5 \rightarrow 3 \rightarrow 6$ & 1 & 3 & 1 & 3 & 2 & 61.5 & 83150 & 26 & $4 \rightarrow 5 \rightarrow 1 \rightarrow 2 \rightarrow 3 \rightarrow 6$ & 2 & 3 & 1 & 1 & 1 & 84.5 & 77450 \\
\hline 8 & $4 \rightarrow 5 \rightarrow 1 \rightarrow 2 \rightarrow 3 \rightarrow 6$ & 1 & 3 & 1 & 3 & 2 & 61.5 & 83150 & 27 & $4 \rightarrow 5 \rightarrow 1 \rightarrow 3 \rightarrow 2 \rightarrow 6$ & 2 & 3 & 1 & 1 & 1 & 84.5 & 77450 \\
\hline 9 & $4 \rightarrow 2 \rightarrow 1 \rightarrow 5 \rightarrow 3 \rightarrow 6$ & 1 & 3 & 1 & 3 & 1 & 63 & 81650 & 28 & $4 \rightarrow 5 \rightarrow 1 \rightarrow 2 \rightarrow 3 \rightarrow 6$ & 1 & 3 & 1 & 1 & 2 & 92 & 76850 \\
\hline 10 & $4 \rightarrow 5 \rightarrow 1 \rightarrow 2 \rightarrow 3 \rightarrow 6$ & 1 & 3 & 1 & 3 & 1 & 63 & 81650 & 29 & $4 \rightarrow 5 \rightarrow 1 \rightarrow 3 \rightarrow 2 \rightarrow 6$ & 1 & 3 & 1 & 1 & 2 & 92 & 76850 \\
\hline 11 & $4 \rightarrow 5 \rightarrow 1 \rightarrow 2 \rightarrow 3 \rightarrow 6$ & 2 & 3 & 1 & 2 & 2 & 67 & 81550 & 30 & $5 \rightarrow 1 \rightarrow 3 \rightarrow 2 \rightarrow 4 \rightarrow 6$ & 1 & 3 & 1 & 1 & 2 & 92 & 76850 \\
\hline 12 & $4 \rightarrow 5 \rightarrow 1 \rightarrow 3 \rightarrow 2 \rightarrow 6$ & 2 & 3 & 1 & 2 & 2 & 67 & 81550 & 31 & $5 \rightarrow 1 \rightarrow 2 \rightarrow 3 \rightarrow 6 \rightarrow 4$ & 1 & 3 & 1 & 1 & 2 & 92 & 76850 \\
\hline 13 & $4 \rightarrow 5 \rightarrow 1 \rightarrow 2 \rightarrow 3 \rightarrow 6$ & 2 & 3 & 1 & 2 & 1 & 68 & 79950 & 32 & $4 \rightarrow 5 \rightarrow 1 \rightarrow 2 \rightarrow 3 \rightarrow 6$ & 1 & 3 & 1 & 1 & 1 & 93 & 75250 \\
\hline 14 & $4 \rightarrow 5 \rightarrow 1 \rightarrow 3 \rightarrow 2 \rightarrow 6$ & 2 & 3 & 1 & 2 & 1 & 68 & 79950 & 33 & $5 \rightarrow 1 \rightarrow 2 \rightarrow 3 \rightarrow 6 \rightarrow 4$ & 1 & 3 & 1 & 1 & 1 & 93 & 75250 \\
\hline 15 & $4 \rightarrow 5 \rightarrow 1 \rightarrow 2 \rightarrow 3 \rightarrow 6$ & 1 & 3 & 1 & 2 & 2 & 75.5 & 79350 & 34 & $5 \rightarrow 1 \rightarrow 3 \rightarrow 2 \rightarrow 4 \rightarrow 6$ & 1 & 3 & 1 & 1 & 1 & 93 & 75250 \\
\hline 16 & $4 \rightarrow 5 \rightarrow 1 \rightarrow 3 \rightarrow 2 \rightarrow 6$ & 1 & 3 & 1 & 2 & 2 & 75.5 & 79350 & 35 & $5 \rightarrow 1 \rightarrow 3 \rightarrow 2 \rightarrow 6 \rightarrow 4$ & 1 & 3 & 1 & 1 & 1 & 93 & 75250 \\
\hline 17 & $5 \rightarrow 1 \rightarrow 2 \rightarrow 3 \rightarrow 6 \rightarrow 4$ & 1 & 3 & 1 & 2 & 2 & 75.5 & 79350 & 36 & $5 \rightarrow 1 \rightarrow 3 \rightarrow 6 \rightarrow 2 \rightarrow 4$ & 1 & 3 & 1 & 1 & 1 & 93 & 75250 \\
\hline 18 & $5 \rightarrow 1 \rightarrow 3 \rightarrow 2 \rightarrow 4 \rightarrow 6$ & 1 & 3 & 1 & 2 & 2 & 75.5 & 79350 & 37 & $5 \rightarrow 1 \rightarrow 4 \rightarrow 2 \rightarrow 3 \rightarrow 6$ & 1 & 3 & 1 & 1 & 1 & 93 & 75250 \\
\hline 19 & $5 \rightarrow 1 \rightarrow 3 \rightarrow 2 \rightarrow 6 \rightarrow 4$ & 1 & 3 & 1 & 2 & 2 & 75.5 & 79350 & & & & & & & & & \\
\hline
\end{tabular}

In this example, the fastest construction time is 52.5 days, and the corresponding minimum cost is 87500 dollars. The slowest construction cost is 93 days, and the corresponding construction cost is 75250 dollars. After performing calculations, the Pareto curve of the knowledge set is obtained. The trend of this curve slopes downwards overall. It is logical that in actual construction, more money must be paid to complete all procedures of all units faster. If the total construction period is extended, the total cost paid will be reduced 
accordingly. From the figure, we can also see that the construction sequence selection and mode selection corresponding to the optimal schedule-cost curve are different. If fixed logic is used, the economic effect must not be achieved. Even the fixed logic cannot satisfy the maximum and minimum constraints of this paper.

\section{CONCLUSION}

This example is a DTCTP problem. The innovations of this article can be divided into 2 parts.

Firstly,a repetitive project construction model of resource continuity under soft logic is proposed. This model is suitable for repetitive construction projects with high idle cost of resources.

Secondly, the minimum and maximum constraints often required in actual engineering are introduced into the model, which has good reference significance for construction projects that require high connectivity between some processes. It helps to avoid construction order and mode selection that do not meet the minimum and maximum constraints.

The following three conclusions can be drawn from the results of TABLE 3 .

The first is construction sequence in the unit does not have a great impact on the total construction period and total cost, which is different from the traditional soft logic construction project, due to the constraint conditions between the second and third processes in this example. It can be seen that multiple construction sequences correspond to the same completion time and the same completion location. In contrast, the choice of construction mode determines the total construction period and total cost to a large extent. In the output results, each different mode selection will correspond to a different total duration and total cost. This is due to the different assumptions of the model in this paper.

According to Table 3, it can be seen that none of the output results are constructed according to fixed logic. Soft logic is more economical than fixed logic when dealing with repetitive projects.

For some construction problems with minimum and maximum constraints, many solutions need to be excluded. The model and algorithm in this paper also have certain reference significance for the project scheduling problem with maximum and minimum constraints.

\section{AUTHORS' CONTRIBUTIONS}

Feng Kong, Chen Yuan contributed to the conception of the study. Kong Feng, Chen Yuan performed the data analyses and wrote the manuscript.

\section{ACKNOWLEDGMENTS}

The authors are very thankful to the editor for his patient reading and valuable suggestions to improve this article.

\section{REFERENCES}

[1] Heravi, G., Moridi, S. Resource-Constrained TimeCost Tradeoff for Repetitive Construction Projects, Springer, Berlin, Heidelberg, 2019, pp. 3265-3274. DOI: https://doi.org/10.1007/s12205-019-0151-X

[2] Sheen G J , Liao L W . A branch and bound algorithm for the one-machine scheduling problem with minimum and maximum time lags[J]. European Journal of Operational Research, 2007, 181(1):102-116.

[3] Xin Z A , Lz B . A constraint programming approach for scheduling repetitive projects with atypical activities considering soft logic[J]. Automation in Construction, 109.

[4] Wang W C. Impact of soft logic on the probabilistic duration of construction projects[J]. International Journal of Project Management, 2005, 23(8):600610.

[5] Kreter, S., Schutt, A. \& Stuckey, P.J. Using constraint programming for solving RCPSP/maxcal. Constraints 22, Springer, Berlin, Heidelberg, 2017, pp. 432-462. DOI: https://doi.org/10.1007/s10601-016-9266-6

[6] Mokhtari, H., Aghaie, A., Rahimi, J. et al. Project time-cost trade-off scheduling: a hybrid optimization approach. Int J Adv Manuf Technol 50, Springer, Berlin, Heidelberg, 2010, pp. 811-822. DOI:https://doi.org/10.1007/s00170010-2543-4

[7] Skutella M. Approximation Algorithms for the Discrete Time-Cost Tradeoff Problem[J]. Mathematics of Operations Research, 1997, 23. 